EPJ Web of Conferences 59, 13010 (2013)

DOI: $10.1051 /$ epjconf/20135913010

(C) Owned by the authors, published by EDP Sciences, 2013

\title{
Determination of self generated magnetic field and the plasma density using Cotton Mouton polarimetry with two color probes
}

\author{
A.S. Joshi ${ }^{\mathrm{a}}$ \\ Laser Plasma Division, Raja Ramanna Centre for Advanced Technology, Indore 452013 , \\ India
}

\begin{abstract}
Self generated magnetic fields (SGMF) in laser produced plasmas are conventionally determined by measuring the Faraday rotation angle of a linearly polarized laser probe beam passing through the plasma along with the interferogram for obtaining plasma density. In this paper, we propose a new method to obtain the plasma density and the SGMF distribution from two simultaneous measurements of Cotton Mouton polarimetry of two linearly polarized probe beams of different colors that pass through plasma in a direction normal to the planar target. It is shown that this technique allows us to determine the distribution of SGMF and the plasma density without doing interferometry of laser produced plasmas.
\end{abstract}

\section{INTRODUCTION}

Self-generated magnetic fields (SGMF) of the order of $100 \mathrm{kG}$ to few MG $[1,2]$ are generated in high density, high temperature laser produced plasmas. The knowledge of SGMF is important as they can significantly inhibit the heat transport $[3,4]$ in the inertial confinement fusion (ICF) plasmas. The conventional optical probe method [1] for the measurement of SGMF requires that along with the Faraday rotation angle (FRA), the plasma density in the probed region of plasma also has to be measured. The plasma density information is obtained from the interferogram formed due to the interference between a reference probe beam and the part of probe beam that passes through the plasma. The main problem in interferometry is that the visibility of the fringes in the interferogram, depends on coherence, which makes it difficult to get plasma density information. The study of SGMF and the knowledge of the plasma density in sub-ps pulse duration is important from point of view of energy transport by the laser pulse in the fast ignition regime [5, 6]. SGMF in plasmas produced by sub-ps laser pulses has been measured by Cotton Mouton polarimetry in the sub-ps time scales [7, 8] using the pump-probe method. However, one does not get information about the plasma density in these measurements done by using only one probe beam. Previously, Joshi et al [9] had proposed a technique for obtaining the plasma density and the SGMF using the FRA measurements of two color probe beams propagating parallel to the planar target through cylindrically symmetric plasmas so that Abel inversion technique could be used for determining the torroidal SGMF and the plasma density. It was shown in this work that the plasma density can be obtained without doing the interferometry, along with the SGMF from the FRA measurements of the two color probes. In this paper, we describe a new method to obtain the information of plasma density and the SGMF using the Cotton Mouton effect based polarimetric ellipticity of the two linearly polarized probe beams having different wavelengths incident normal to the target. This technique allows one to calculate the SGMF and the plasma density without the need of

ae-mail: asjoshi@rrcat.gov.in

This is an Open Access article distributed under the terms of the Creative Commons Attribution License 2.0, which permits unrestricted use, distribution, and reproduction in any medium, provided the original work is properly cited. 


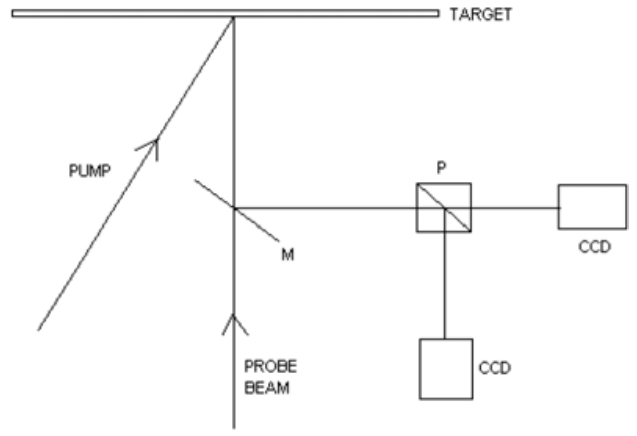

Figure 1. Typical schematic diagram of the set-up.

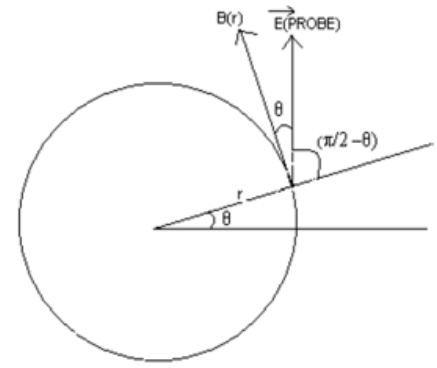

Figure 2. The direction of SGMF and the electric field of the probe beam.

interferometry. The proposed technique can also be used to study the evolution of magnetic fields and the plasma density in femtosecond laser produced plasma.

\section{DETAILS OF THE TECHNIQUE}

Consider plasma that is produced by a pump beam at an angle that is different than the normal to the target surface as shown in the Figure 1. A high contrast polarizer (typically 1:1000 or more) can be used to measure the ellipticity of the polarized beam reflected by the plasma. Two identical, high dynamic range CCD cameras can be used as detectors of probe beams' relative intensity in two orthogonal polarizations at different locations. A practical way of getting the desired probe beams, with suitable properties has been reported earlier [10].

Two probe beams are incident on the target along the target normal as shown in the Figure 1 . The wavelengths of both the probe beams are smaller than that of the pump beam for probing the critical density region of plasma where maximum SGMF exists. It is assumed that the size of plasma is small, thereby the Z-coordinate of the two critical density surfaces for the two probe beams are close enough and assumed to be same i. e. $\mathrm{z}_{\mathrm{c}}$. It is assumed that the plasma is cylindrically symmetric hence it produces torroidal magnetic field parallel to the target surface. In this geometry, the direction of propagation of the wave vector of the probe beams is perpendicular with respect to the planar target and hence the magnetic field as well. So the rotation of polarization of the probe beam due to Faraday Effect does not take place. In general, the Faraday rotation angle $\alpha$ and the ellipticity $\mathrm{E}$ (that is ratio of major and minor axis of the elliptical polarization) of a probe beam on propagation through a small thickness in $\mathrm{Z}$ direction of the plasma is given by [11]:

$$
\mathrm{d} \alpha(\mathrm{r}, \theta, \mathrm{z})=\frac{X Y \cos \Phi}{2\left(1-Y^{2}\right)} \frac{w}{c} \mathrm{dz} \quad d E(\mathrm{r}, \theta, \mathrm{z})=\left|\frac{X Y^{2} \sin ^{2} \Phi}{2\left(1-Y^{2}\right)} \sin (2 \beta) \frac{w}{c} \mathrm{dz}\right|
$$

where $\mathrm{X}=\left(\omega_{\mathrm{p}} / \omega\right)^{2}, \mathrm{Y}=\Omega / \omega, \Omega=\mathrm{eB}(\mathrm{r}) / \mathrm{m}, \omega_{\mathrm{p}}=\left[\mathrm{n}(\mathrm{r}, \mathrm{z}) \mathrm{e}^{2} / \varepsilon_{0} \mathrm{~m}\right]^{0.5}$ is the plasma frequency, $\omega$ is the frequency of the probe laser beam and e and $\mathrm{m}$ are the charge and mass of electron. Here $\mathrm{B}(\mathrm{r})$ is the magnitude of the torroidal magnetic field experienced by the probe beams all along the distance ' $\mathrm{dz}$ ' of plasma. Similarly, $\mathrm{n}(\mathrm{r}, \mathrm{z})$ is the density of the cylindrically symmetric plasma experienced by the probe beams that propagate in the Z-direction. $\Phi$ is the angle between the magnetic field and the wave vector of the probe beams? Since the torroidal magnetic field is perpendicular to the propagation direction of the probe beam, $\cos \Phi=0$ and $\sin \Phi=1$. Thus, as expected, the value of the FRA $\alpha$ is zero in our chosen geometry. Hence, in this direction, the contribution to the polarization rotation comes entirely from the ellipticity of the polarization on propagation through the magnetized plasma due to Cotton Mouton 
effect. The angle $(\pi / 2)-\beta$ has been shown to be the angle between the direction of polarization of the incident probe beam and the magnetic field [11]. Figure 2 above, shows that the value of angle $\beta$ can now be obtained in terms of cylindrical angular co-ordinate $\theta$ of the probe beam as $\beta=(\pi / 2)-\theta$. Due to normal incidence of the probe beams, the relation between $\beta$ and $\theta$ remains un-altered all along the $\mathrm{z}$ axis as there are no plasma refraction effects deviating the beams. Substituting for the value of $\beta$ in equation (1), one gets equation (2).

$$
d E(r, \theta, \mathrm{z})=\left|\frac{X Y^{2}}{2\left(1-Y^{2}\right)} \sin (2 \theta) \frac{w}{c} d z\right| .
$$

To obtain the plasma density and SGMF distribution from the measured ellipticity, it is assumed that the probe beams have sizes comparable or more than that of plasma. A spatially resolved measurement of the ellipticity at different $(r, \theta)$ locations gives the values of the plasma density and SGMF at those $(r, \theta)$ values. It is assumed that the plasma density can be expressed as $n(r, z)=n(r) n(z)$ and that there is no $\theta$ dependence as the plasma is cylindrically symmetric. By considering a self-similar expansion of the plasma in vacuum, $n(z)$ is given by $n(z)=n_{c} \exp \left(-\left(z-z_{c}\right) / c_{s} t\right)$ [12], where $n_{c}$ is the plasma critical density, $\mathrm{z}_{\mathrm{c}}$ is the Z-coordinate of the critical density surface. Here, $\mathrm{c}_{\mathrm{s}}=\left(\mathrm{ZkT}_{\mathrm{e}} / \mathrm{m}_{\mathrm{i}}\right)^{0.5}$ is the ion speed and $t$ is the time of evolution of the plasma. While deriving equation 2 , it is assumed that the phase change for the orthogonal polarizations is small because of small size of the plasma and rate of change of plasma refractive indices is small for both the orthogonal polarizations [11]. Due to normal incidence of the probe beams, the Fresnel reflection coefficient for the electric fields of both the orthogonal polarizations is the same as the probe beams are incident in direction parallel to axis of cylindrically symmetric plasma. In this direction, the refractive indices are same for both the polarizations. Hence, for normal incidence, the ellipticity (which is ratio of amplitudes of the orthogonal polarizations) acquired by the beam because of magnetic field is same for the transmitted and the reflected beam at any value of $\mathrm{Z}$-coordinate. Hence, integrating from $\mathrm{z}$ to $\mathrm{z}_{\mathrm{c}}$ and back one gets the ellipticity of the reflected beam (that goes to the CCD detector as shown in Figure 1) to be:

$$
E(r, \theta)=\left|\frac{S Y^{2}}{\left(1-Y^{2}\right)} \sin (2 \theta) \frac{w}{c}\right|
$$

where $\mathrm{S}=\mathrm{n}_{c}\left\{1-\exp \left(\mathrm{z}_{\mathrm{c}} / c_{\mathrm{s}} \mathrm{t}\right)\right\} \mathrm{c}_{\mathrm{s}} \mathrm{t}\left[\mathrm{n}_{\mathrm{e}}(\mathrm{r}) \mathrm{e}^{2} / \varepsilon_{0} \mathrm{~m}\right] / \omega^{2}$. For $\mathrm{c}_{\mathrm{s}} t \gg \mathrm{z}_{\mathrm{c}}, \mathrm{S}$ is positive. It can be seen from equation 3 that the ellipticity of the polarization depends on the frequency of the probe laser. Let $\mathrm{E}_{1}(\mathrm{r}, \theta)$ be the ellipticity of the first probe beam and $\mathrm{E}_{2}(\mathrm{r}, \theta)$ be the ellipticity of the second probe beam that is measured at different values of $r$ and $\theta$ and as measured by the detectors shown in the Figure 1. Let $n_{1}$ and $\mathrm{n}_{2}$ be the critical densities for the two probe beams. As the size of plasma is assumed to be small, the $\mathrm{z}$ coordinates of the plasma critical densities of the two probe beams can be treated to be equal and occurring at $\mathrm{z}_{\mathrm{c}}$. Under this assumption, the coefficients $\mathrm{Y}$ and $\mathrm{S}$ differ only because of frequencies $\left(\mathrm{w}_{1}\right.$ and $\mathrm{w}_{2}$ of the probe beams. Let $\mathrm{Y}_{1}(\mathrm{r})=\Omega / \omega_{1}$ and $\mathrm{S}_{1}(\mathrm{r})$ be the coefficients for the first probe beam, and $\mathrm{Y}_{2}(\mathrm{r})=\Omega / \omega_{2}$ and $\mathrm{S}_{2}(\mathrm{r})$ be the coefficients for the second probe beam. Taking the ratios of the ellipticities and substituting for corresponding values of $\mathrm{S}$ and $\mathrm{Y}$, we get:

$$
\frac{\mathrm{E}_{1}(\mathrm{r}, \theta)}{\mathrm{E}_{2}(\mathrm{r}, \theta)} \sim \mathrm{C}(\mathrm{r})=\frac{\mathrm{n}_{1} w_{2}\left(w_{2}^{2}-\Omega^{2}\right)}{\mathrm{n}_{2} w_{1}\left(w_{1}^{2}-\Omega^{2}\right)} .
$$

The value of the magnitude of torroidal magnetic field can be found by substituting the experimentally measured value of $\mathrm{C}(\mathrm{r})$ and substituting for $\Omega$ in the above equation.

$$
\mathrm{B}^{2}(\mathrm{r})=\frac{\mathrm{n}_{1} w_{2}^{3}-\mathrm{n}_{2} \mathrm{C}(\mathrm{r}) w_{1}^{3}}{\mathrm{n}_{1} w_{2}-\mathrm{n}_{2} w_{1} \mathrm{C}(\mathrm{r})} \frac{\mathrm{m}^{2}}{\mathrm{e}^{2}} .
$$

The value of $Y_{1}(r)$ and $Y_{2}(r)$ can now be calculated knowing the value of magnitude $B(r)$ of SGMF. To calculate the plasma density $n(r)$, consider the ellipticity of polarization for both the probe beams at 
same co-ordinate $(\mathrm{r}, \theta)$.

$$
\begin{aligned}
& E_{1}(r, \theta)=\left|S_{1} \sin (2 \theta) \frac{w}{c}\left[\frac{1}{\left(1-Y_{1}^{2}(r)\right)}-1\right]\right|=\left|K_{1}\left(w_{1}\right) n(r)\left[\frac{1}{\left(1-Y_{1}^{2}(r)\right)}-1\right]\right| \\
& E_{2}(r, \theta)=\left|S_{2} \sin (2 \theta) \frac{w}{c}\left[\frac{1}{\left(1-Y_{2}^{2}(r)\right)}-1\right]\right|=\left|K_{1}\left(w_{2}\right) n(r)\left[\frac{1}{\left(1-Y_{2}^{2}(r)\right)}-1\right]\right|
\end{aligned}
$$

where $\mathrm{K}_{1}\left(\omega_{1}\right)=\left(\mathrm{n}_{1}\left\{1-\exp \left(\mathrm{z}_{\mathrm{c}} / \mathrm{c}_{\mathrm{s}} \mathrm{t}\right)\right\} \mathrm{c}_{\mathrm{s}} \mathrm{t}\left[\mathrm{e}^{2} / \varepsilon_{0} \mathrm{~m}\right] / \mathrm{c} \omega_{1}\right) \sin (2 \theta)$ and $\mathrm{K}_{1}\left(\omega_{2}\right)=\left(\mathrm{n}_{2}\left\{1-\exp \left(\mathrm{z}_{\mathrm{c}} / \mathrm{c}_{\mathrm{s}} \mathrm{t}\right)\right\} \mathrm{c}_{\mathrm{s}} \mathrm{t}\right.$ $\left.\left[\mathrm{e}^{2} / \varepsilon_{0} \mathrm{~m}\right] / \mathrm{c} \omega_{2}\right) \sin (2 \theta)$. Care has to be taken in choosing the sign of $\mathrm{K}_{1}$ and $\mathrm{K}_{2}$ appropriately in the above expression so as to make $E_{1}$ and $E_{2}$ positive. The value of the plasma density can be evaluated as:

$$
\mathrm{n}(\mathrm{r})=\frac{\frac{\mathrm{E}_{1}\left(w_{1}\right)}{\mathrm{K}_{1}\left(w_{1}\right)}-\frac{\mathrm{E}_{2}\left(w_{2}\right)}{\mathrm{K}_{1}\left(w_{2}\right)}}{\frac{1}{1-Y_{1}^{2}}-\frac{1}{1-Y_{2}^{2}}} .
$$

Thus it is seen that to obtain the distributions of two measurables; namely the magnitude of SGMF and the plasma density, the measured ellipticities of the two probe beams are sufficient.

\section{CONCLUSIONS}

A new method for the measurement of the self generated magnetic field and the density of laser produced plasma using two linearly polarized probe beams of different colors for the measurement of the polarization ellipticity because of Cotton Mouton effect on propagation through plasma is proposed. It is shown that from the measured ellipticities at different $r$ and $\theta$, the distribution of plasma density and the self generated magnetic field can be calculated for the cylindrically symmetric plasmas. The advantage of this method over the conventional method of measuring SGMF by using polaro-interferometery is that the problem of poor fringe visibility in some parts of the interferogram (which is invariably present) can be by-passed and distribution of plasma density and SGMF can be measured .

\section{References}

[1] J. A. Stamper, B. H. Ripin, Phys. Rev. Lett. 34, 138 (1975)

[2] A. Raven, O. Willi, P. T. Rumsby, Phys. Rev. Lett. 41, 554 (1978)

[3] D. S. Montgomery, O. L. Landen, R. P. Drake, K. G. Estabrook, H. A. Baldis, S. H. Batha, K. S. Bradley, R. J. Procassini, Phys. Rev. Lett. 73, 2055 (1993)

[4] C. A. Back, D. H. Kalantar, R. L. Kauffman, R. W. Lee, B. J. MacGowan, D. S. Montgomery, L. V. Powers, Phys. Rev. Lett. 77, 4350 (1996)

[5] T. Lehner, Europhysics Lett. 50, 480 (2000)

[6] M. Tabak et al, Phys. Plasmas 1, 1626 (1994)

[7] A. S. Sandhu, A. K. Dharmadhikari, P. P. Rajeev, G. R. Kumar, S. Sengupta, A. Das, P. K. Kaw, Phys. Rev. Lett. 89, 225002 (2002)

[8] S. Kahaly, S. Mondal, G. Ravikumar, S. Sengupta, A. Das, Phys. Plasmas 16, 043114 (2009)

[9] A. S. Joshi, P. A. Naik, S. Barnwal, Y. B. S. R. Prasad, Opt. Commun. 283, 4713 (2010)

[10] M. P. Kamath, A. P. Kulkarni, S. Jain, P. K. Tripathi, A. S. Joshi, P. A. Naik and P. D. Gupta, Pramana J. Physics 75, 883 (2010)

[11] I. H. Hutchinson, Principles of Plasma Diagnostics, pp. 137-140, (Cambridge University Press, Cambridge, UK, Second edition, 2002)

[12] W. L. Kruer, Physics of Laser Plasma interactions, (Addison-Wesley, New York, 1988) 